\title{
Miniaturized EAPs with compliant electrodes fabricated by ion implantation
}

\author{
H. Shea* \\ Ecole Polytechnique Fédérale de Lausanne (EPFL), Rue Jaquet Droz 1, CH-2002 Neuchatel, \\ Switzerland
}

\begin{abstract}
Miniaturizing dielectric electroactive polymer (EAP) actuators will lead to highly-integrated mechanical systems on a chip, combining dozens to thousands of actuators and sensors on a few $\mathrm{cm}^{2}$. We present here $\mu \mathrm{m}$ to $\mathrm{mm}$ scale electroactive polymer (EAP) devices, batch fabricated on the chip or wafer scale, based on ion-implanted electrodes. Low-energy (2-10 keV) implantation of gold ions into a silicone elastomer leads to compliant stretchable electrodes consisting of a buried $20 \mathrm{~nm}$ thick layer of gold nanoparticles in a silicone matrix. These electrodes: 1) conduct at strains up to $175 \%, 2$ ) are patternable on the $\mu \mathrm{m}$ scale, 3) have stiffness similar to silicone, 4) have good conductivity, and 5) excellent adhesion since implanted in the silicone. The EAP devices consist of 20 to $30 \mu \mathrm{m}$ thick silicone membranes with $\mu \mathrm{m}$ to $\mathrm{mm}$-scale ion-implanted electrodes on both sides, bonded to a holder. Depending on electrode shape and membrane size, several actuation modes are possible. Characterization of $3 \mathrm{~mm}$ diameter bi-directional buckling mode actuators, mm-scale tunable lens arrays, 2-axis beam steering mirrors, as well as arrays of 72 cell-size $\left(100 \times 200 \mu \mathrm{m}^{2}\right)$ actuators to apply mechanical strain to single cells are reported. Speeds of up to several $\mathrm{kHz}$ are observed.
\end{abstract}

Keywords: EAP, electroactive polymer, MEMS, ion implantation

\section{INTRODUCTION}

The term "artificial muscles" has been coined to describe a range of electroactive polymer (EAP) actuators with remarkable muscle-like properties: high flexibility, high strain (over 100\%), and high energy density ${ }^{[1],[2],[3]}$. Figure 1 shows schematically one type of EAP, the dielectric elastomer actuator, which consists a soft elastomer sandwiched between two compliant electrodes, generally made of carbon powder or carbon greases. When a voltage is applied between the electrodes an electrostatic force is generated, compressing the elastomer, which expands in-plane. Depending on the boundary conditions, many different actuators and sensors can be made, from large active fins for blimps $^{[6]}$, to haptic systems, tunable optics ${ }^{[4]}$, to $\mathrm{cm}$-scale walking, grasping and swimming $\operatorname{robots}^{[1],[2]}$.
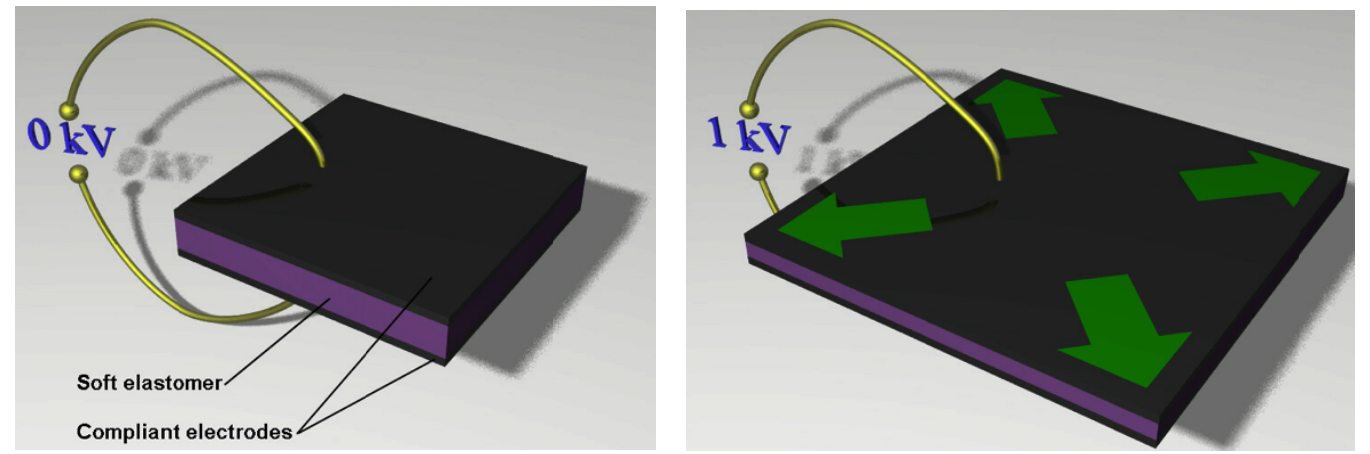

Figure 1: basic principle of dielectric elastomer actuators. When a high voltage is applied between the flexible electrodes, the electrostatic pressure compresses the elastomer. Removing the voltage returns the device to its initial configuration. From ref [9].

* herbert.shea@epfl.ch; http://lmts.epfl.ch

Electroactive Polymer Actuators and Devices (EAPAD) 2011, edited by Yoseph Bar-Cohen, Federico Carpi, Proc. of SPIE Vol. 7976, 79760R · @ 2011 SPIE · CCC code: 0277-786X/11/\$18 · doi: 10.1117/12.882212 
Miniaturizing EAP devices is challenging because the compliant electrodes must a) conduct well at strains of over $30 \%$, b) yet be softer than the elastomers to allow for large mechanical deformation, and c) be easily patterned on the micron scale so that $\mu \mathrm{m}$ scale devices can be realized.

Some groups have succeeded in patterning carbon powder on the $\mu \mathrm{m}$ scale, for instance Aschwanden et al. ${ }^{[4]}$ report 100 $\mu \mathrm{m}$ wide electrodes. The seemingly obvious solution of thin metal traces is very challenging as such metal lines break at only $3 \%$ strain and greatly stiffen the device, since most metals used in microfabrication have Young's modulii 5 orders of magnitude larger than silicone elastomers. Pimpin et al. ${ }^{[5]}$ have patterned concentric metal traces on PDMS, and obtained devices that operated well but whose deflection was limited by the stiffness of the metal electrode. Creating nanocomposite is another possible route: the use of nanoporous gold films have been reported, allowing up to $25 \%$ strain ${ }^{[7]}$, and electrodes based on the precipitation and diffusion of nm-sized platinum particles ${ }^{[8]}$.

We summarize in this paper the work we have done to batch fabricating arrays of miniaturized polymer actuators based on highly stretchable electrodes made by low-energy ion-implantation into the elastomer ${ }^{[13]}$. Implanting gold ions into silicone rubbers forms a 20-50 nm thick nanocomposite, consisting of gold particles in the silicone matrix. The gold nanoparticles conduct by ohmic contact between particles, but can slide with respect to each other, allowing conduction at up to $175 \%$ strain, while adding very little stiffness to the actuator ${ }^{[15]}$.

In section 2 we present the ion-implanted electrodes: their fabrication, and mechanical and electrical properties. In section 3 we present several EAP devices made using these ion-implanted electrodes, from simple buckling mode devices to arrays of cell-stretching actuators.

\section{ION-IMPLANTED COMPLIANT ELECTRODES}

We present here a technique to make a nanocomposite conductor, consisting of gold nanoparticles in a polydimethylsiloxane (PDMS) matrix. Rather than mixing gold particles with uncured PDMS, or depositing a powder of nanoparticles on the surface of the PDMS, we have found that low-energy (less than $10 \mathrm{keV}$ ) implantation of gold ions into PDMS leads to a $50 \mathrm{~nm}$ thick nanocomposite of gold particles of size 2-20nm in the PDMS. The underlying PDMS is unaffected, thus maintaining its excellent properties for use in EAPs.

Figure 2 is a TEM cross-section image showing the gold-PDMS nanocomposite on top of the virgin PDMS. The size of the particles depends on ion dose and on ion energy, as described in detail in ref [15]. Figure 3 shows the dependence of the gold particle size on ion energy and on the amount of gold in the nanocomposite layer, expressed as a volume fraction.

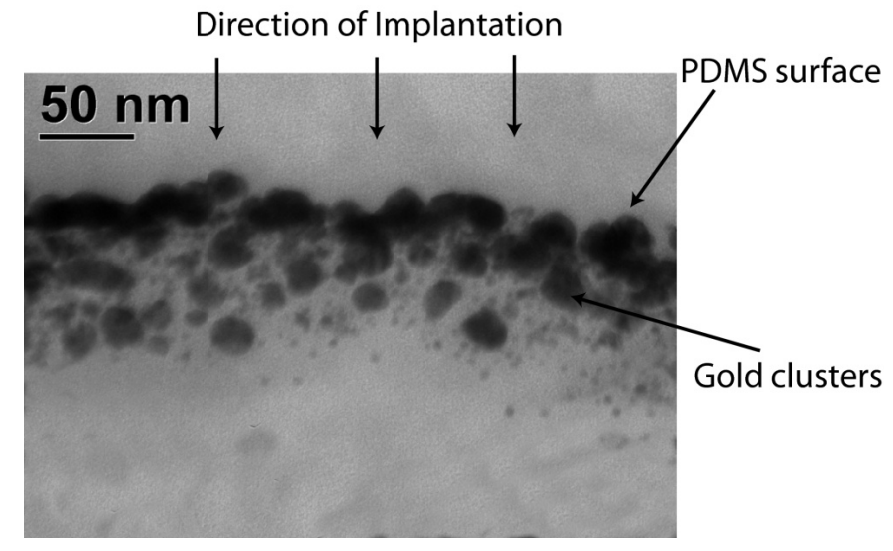

Figure 2: TEM cross-section of gold-ion implanted PDMS (5 keV ion energy). The gold nanoparticles are the back circular objects just below and at the surface of the PDMS. From ref [13] based on a sample preparation technique reported in [14].

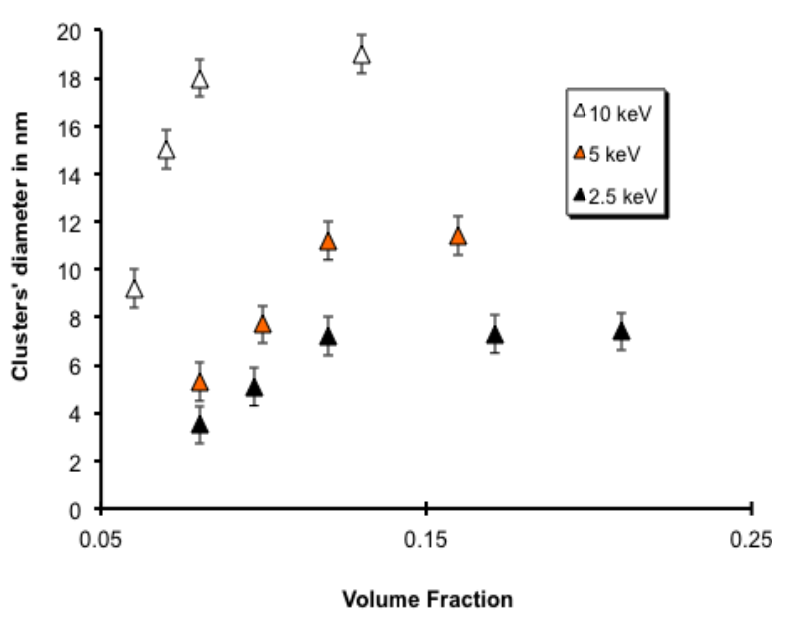

Figure 3: Average particle size as a function of gold ion energy. The volume fraction refers to the gold volume fraction in the nanocomposite film (40 nm thick), not in the entire PDMS layer ( $25 \mu \mathrm{m}$ thick). 


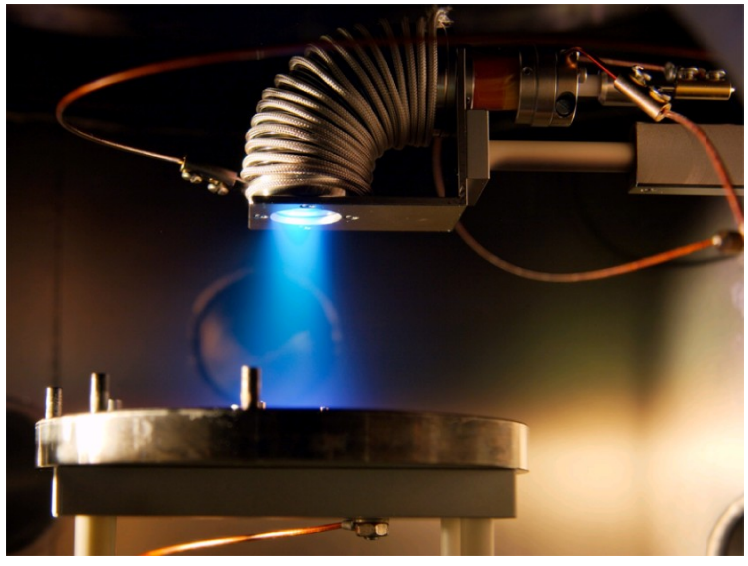

Figure 4: Photograph of the FCVA ion source during one pulse. The substrate holder (bottom disk with vertical pins) can be biased up to $-5 \mathrm{kV}$, image from ref [15].

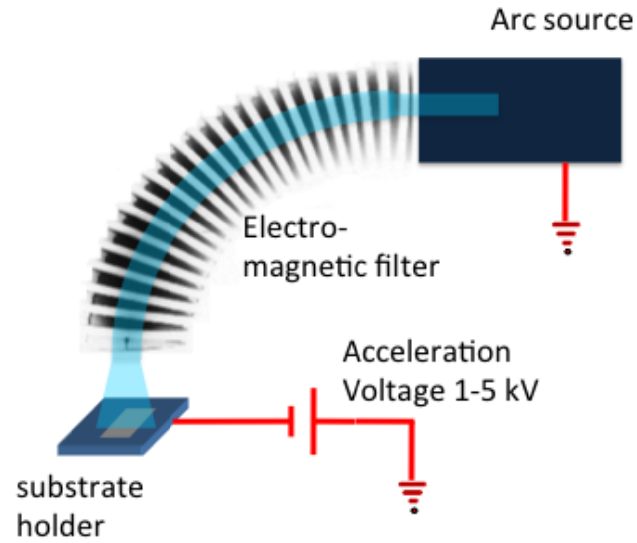

Figure 5: Simplified schematic of the FCVA source.

An ion dose of order $10^{16}$ ions $/ \mathrm{cm}^{2}$ is required to reach sufficient conductivity. In order to achieve this fluence in a short time, a Filtered Cathodic Vacuum Arc (FCVA) ion source was used, which allows the desired surface resistivity of 1 $\mathrm{k} \Omega$ /square to be achieved in a few minutes. The FCVA is a pulsed source, essentially a plasma gun that emits doublycharged ions at an energy of order $50 \mathrm{eV}$, and incorporates at $90^{\circ}$ bend that acts as a filter to remove macroparticles from the arc source (see Figure 4 and Figure 5). A key element is a 0 to $-5 \mathrm{kV}$ bias on the sample holder to accelerate ions in order to implant them rather than simply deposit a metallic film. Our implanter ${ }^{[1]][15]}$ is based on an RHK Technology ARC20 source, and the $1 \mathrm{~cm}^{2}$ spot can be rastered over a $100 \mathrm{~mm} \times 100 \mathrm{~mm}$ area.

The implantations are performed through a laser cut steel shadow mask ${ }^{[13]}$. Since the metal ions are implanted they adhere to any surface, allowing 3D patterning of electrodes, for instance enabling electrodes to be pattered on the sidewalls of PDMS channels for microfluidics ${ }^{[16]}$. The resolution is currently limited to $50 \mu \mathrm{m}$ using steel shadow masks; photolithography would enable $\mu \mathrm{m}$ features, and we are investigating stencil-masks for sub- $\mu \mathrm{m}$ devices.

There is a tradeoff between the conductivity of the implanted layer and its stiffness. The higher the implanted doses, the lower the resistivity, but the higher the stiffness. For EAP application, ideally there would be no stiffening of the elastomer by the electrodes. For flexible electronics applications, the stiffness may not be of concern. Since we focus here on EAP devices, a major consideration is finding the ion dose that will lead to minimal stiffening of the PDMS layer while providing high enough conductivity that the RC time constant of the device will be much shorter than the mechanical time constant.

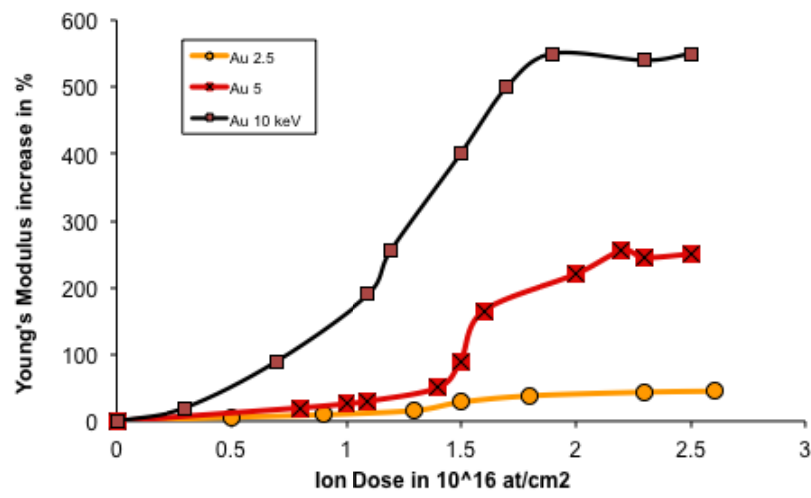

Figure 6: Percentage increase in the effective Young's modulus of a $25 \mu \mathrm{m}$ thick Sylgard 186 PDMS film following gold ion implantation, as a function of ion dose for three ion energies.

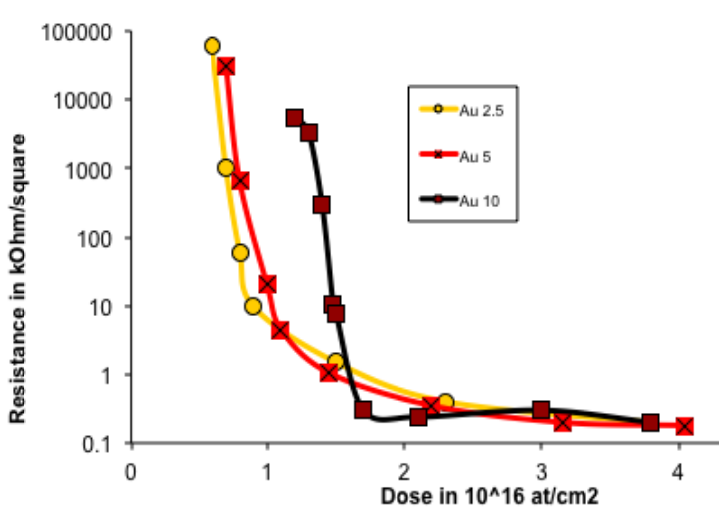

Figure 7: Surface resistivity of the Au-PDMS nanocomposite as a function of gold ion dose for three ion energies. 
The PDMS used for most of our work is Sylgard 186, which has a Young's modulus of 0.8 MPa. A bulge test technique is used to measure the effective Young's modulus of the composite consisting of the $50 \mathrm{~nm}$ thick nanocomposite and of the underlying $25 \mu \mathrm{m}$ thick PDMS film. This is plotted in Figure 6 as a function of gold ion dose. Figure 7 is a plot of surface resistivity as a function of ion dose. Further details can be found in reference [15]. A convenient way to determine the optimum ion dose is to plot the relative increase in Young's modulus as a function of the film resistivity, as done in Figure 8 for three different ions types.

Another key parameter is the ability of the implanted film to conduct at large strain, and to do so after millions of cycles. Figure 9 is a plot vs. applied uniaxial strain of the ratio of resistivity when strained to the initial resistivity ${ }^{[13]}$. Each plot ends at the strain at which the film ceases to conduct. For films with lower implanted doses, the films can be stretched to more than double its initial length and still conduct. This behavior also allows the use the implanted electrodes as strain sensors (piezo-resistors). Electrodes cyclically cycled to $30 \%$ strain remained operational after more than $10^{5}$ cycles ${ }^{[13]}$.

We investigated the effect of gamma and proton irradiation on the optical and mechanical properties of gold-ion implanted PDMS, as reported in [18]. The optical transmission is not strongly affected, but the stiffness of the PDMS roughly doubles after $100 \mathrm{krad}$ of Co60 gamma irradiation and after $1 \mathrm{Mrad}$ (TID) of $3.5 \mathrm{MeV}$ protons, which must be taken in account for possible space applications.

In conclusion, gold-ion implanted PDMS electrodes can: 1) conduct at strains up to 175\%,2) are patternable on the $\mu \mathrm{m}$ scale, 3) add little stiffness to the silicone, 4) have good conductivity allowing for fast actuation, and 5) offer excellent adhesion since they are implanted.

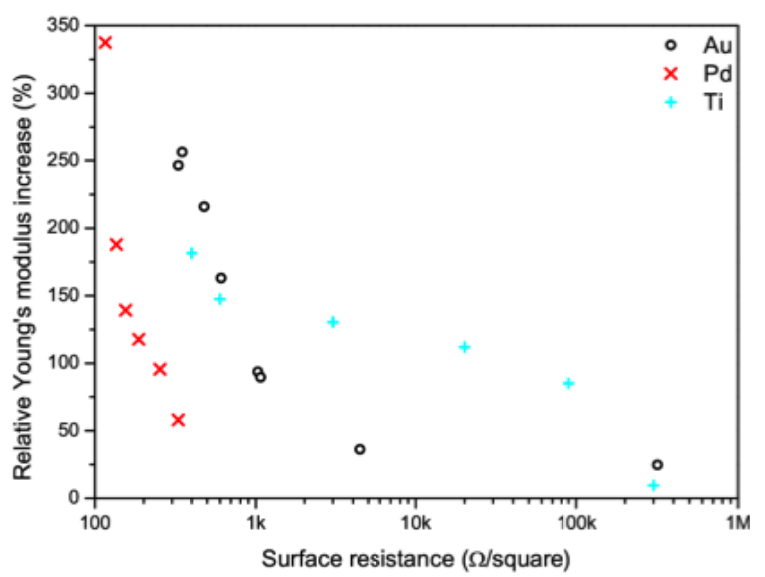

Figure 8: Percentage increase in the effective Young's modulus of a $25 \mu \mathrm{m}$ thick Sylgard 186 PDMS film vs. surface resistivity, showing the trade-off between low stiffness and low resistance.

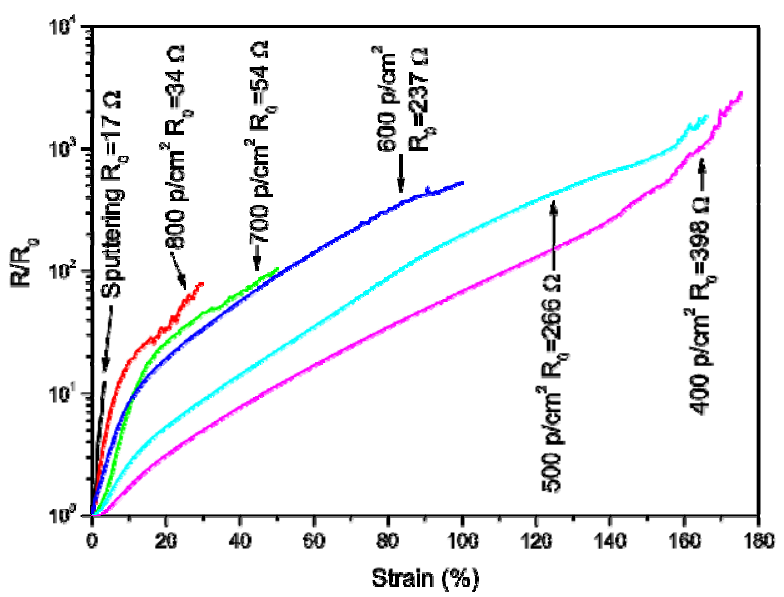

Figure 9: Relative change in surface resistivity vs. applied uniaxial strain for gold implanted PDMS. The lower the implanted dose, the larger the maximum strain at which the film still conducts, from ref [13].

\section{ACTUATORS}

The compliant electrodes described in the previous section were used to fabricate various EAP devices. The overall aim is to batch fabricate arrays of micro-actuators, with critical dimensions ranging from $50 \mu \mathrm{m}$ to $5 \mathrm{~mm}$.

\subsection{Buckling mode actuators}

The first devices we reported based on ion-implantation were $3 \mathrm{~mm}$ diameter buckling mode actuators ${ }^{[10]}$. Figure 10 is a schematic cross-section of the device concept, which consists of a $20 \mu \mathrm{m}$ thick PDMS membrane with patterned ionimplanted electrodes on both sides bonded to a Pyrex chip with powder-blasted through-holes. A finished chip with 4 devices can be seen in Figure 13. When a voltage is applied to the electrodes, the film is compressed (Maxwell pressure), and hence expands in plane. The boundary conditions (PDMS-glass bonding) prevent motion of the periphery, and so the 
membrane buckles up or down, as in Figure 12. A detailed process flow for devices with reaction time limited only by the device mechanics and not by any electrical contract issues is reported in [11].

The devices served initially as a test of the feasibility of using ion implantation for making electrodes for EAPs, and of the maximum displacement that could be achieved. The unloaded vertical deflection of three devices of different diameters is plotted in Figure 14 as a function of applied voltage. The maximum displacement is limited by dielectric breakdown, and corresponds to over $25 \%$ of the device diameter, a much larger percentage displacement than can be achieved with silicon MEMS. The percentage displacement is comparable with macroscopic (cm-scale) buckling mode actuators, showing the implanted electrodes allow for good performance (minimal stiffening) in addition to excellent clean-room compatibility and $\mu \mathrm{m}$-scale patternability.

The device speed was measured with a laser Doppler vibrometer, and shows underdamped behavior in air. The device dynamics depend on its resonance frequency, with very little viscoelastic effects ${ }^{[11]}$. The 2 to $3 \mathrm{~mm}$ diameter membranes have first out-of-plane frequencies of order 1 to $3 \mathrm{kHz}$, depending on stiffness and initial stress, which can be tuned by choice of the curing conditions. The resonance frequency (and hence compliance) can be electrically tuned from the initial value down to zero at the buckling threshold ${ }^{[12]}$, allowing possible applications in acoustic filtering.

These PDMS-based actuators exhibit very long lifetime: several devices were actuated to over 2 million cycles with no measureable change in properties. This is not surprising for non-prestretched silicone-based device operated in the elastic regime.

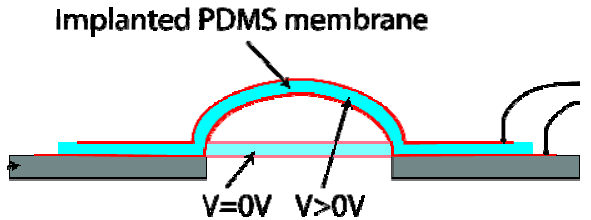

Figure 10: schematic cross-section of a buckling-mode dielectric elastomer actuator. The implanted electrodes are on the top and bottom of the elastomer.

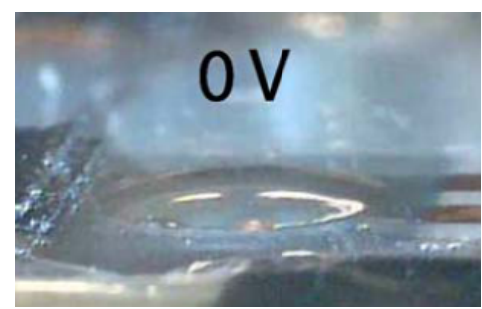

Figure 11: Buckling mode actuator at $0 \mathrm{~V}$, same device as in Figure 13. Membrane diameter is $3 \mathrm{~mm}$.

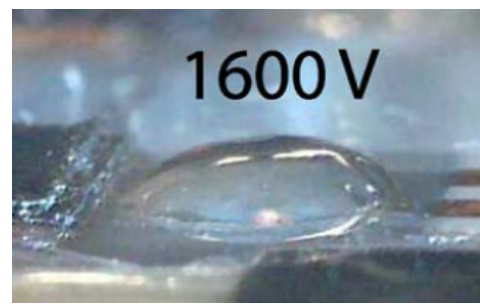

Figure 12: Buckling mode actuator at $1600 \mathrm{~V}$. The implanted electrodes are barely visible, being nearly transparent.

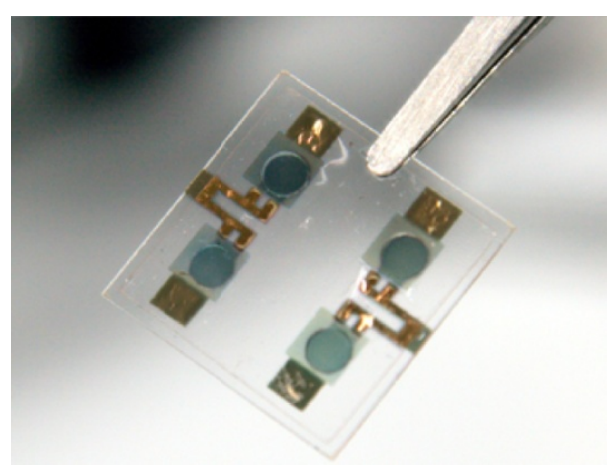

Figure 13: 4 buckling-mode actuators made of ionimplanted PDMS bonded to a glass chip. Device diameter is 2 and $3 \mathrm{~mm}$. Ion implanted region are gray, gold contact pads are gold. From ref [11].

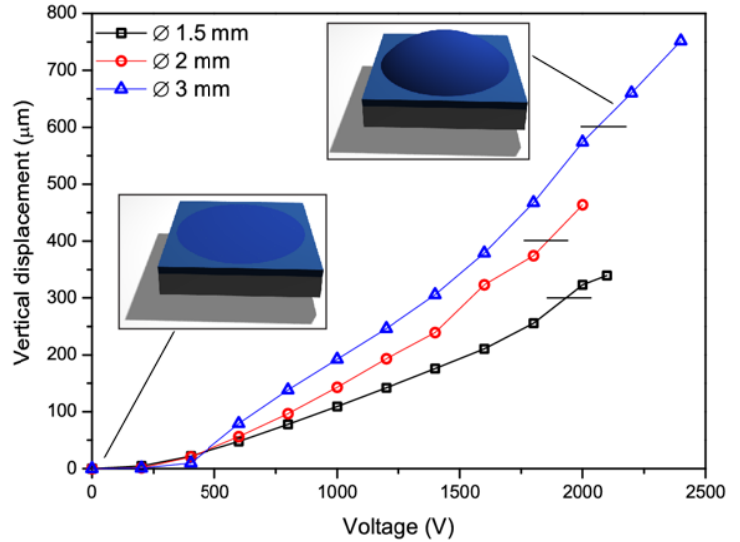

Figure 14: Measured displacement vs. actuation voltage for three EAP devices, showing vertical displacements of over $50 \%$ of the device radius. 


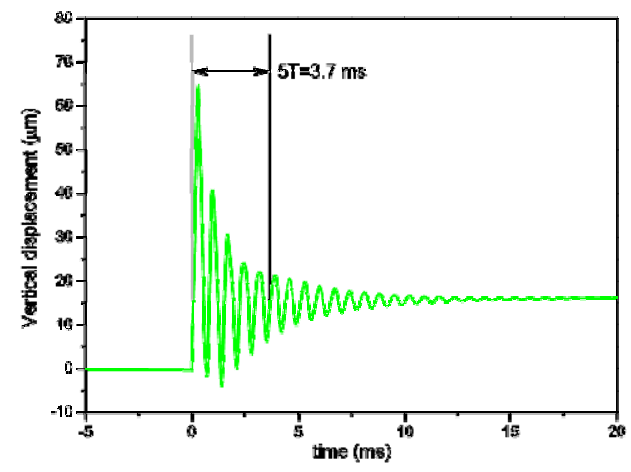

Figure 15: Measured response of the device to a voltage step showing underdamped behavior and a resonance frequency of $1.3 \mathrm{kHz}$. From ref [11].

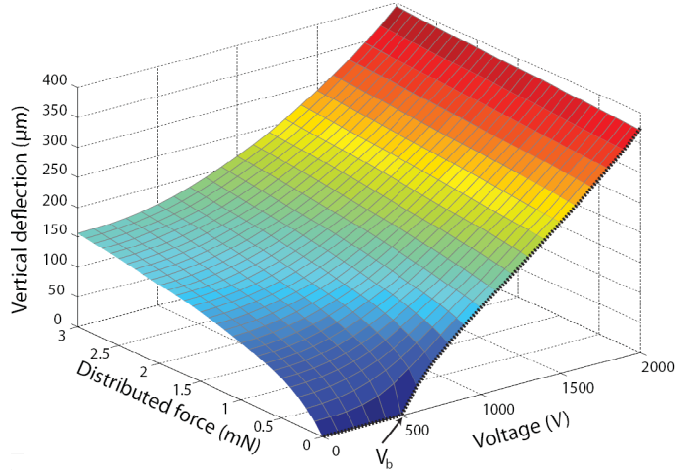

Figure 16: Theoretical vertical displacement of a $2 \mathrm{~mm}$ diameter $20 \mu \mathrm{m}$-thick membrane with $\mathrm{Y}=2 \mathrm{MPa}$, and $\sigma=20 \mathrm{kPa}$ From ref. [11].

\subsection{Applications of the buckling-mode actuator}

\section{Tunable lenses}

Buckling-mode actuators can be used as actuators for tip/tilt/piston platforms, as pumps and active valves in microfluidic circuits, as tunable lenses, etc. A model was developed ${ }^{[\rho]}$ to predict loaded displacement as a function of device geometry and membrane properties, as illustrated in Figure 16, allowing devices to be dimensioned for different possible applications. Our focus is on miniaturized devices, allowing many to be simultaneously fabricated on one chip.

We developed several techniques to make tunable lenses, based on the principle illustrated in Figure 17. When a voltage is applied to the "active" membrane, it expands in area, thus increasing its curvature, and the fluidically coupled "passive" membrane, used as a lens, decreases its curvature, hence increases its focal length. In a first generation ${ }^{[18]}$, we used ion-implantation to make the electrical contacts on both sides of the membrane, but we were then we able to replace one implanted contact by using a conductive liquids as the optical fluid. Ionic liquids are particularly well suited to this task, as they have immeasurably small vapor pressure at room temperature, and hence do not evaporate, and offer very high conductivity. Figure 18 is a simplified diagram of the assembly of a 2 tunable lenses, and 2 actuators. Using a control voltage from 0 to $1.7 \mathrm{kV}$ we demonstrated a tuning range from $4 \mathrm{~mm}$ to $8 \mathrm{~mm}$ for a $2 \mathrm{~mm}$ diameter lens. The operating point is set in large part by the initial pressure of the liquid.

Tuning speed is of order 1 second, limited for now by the stiffness of the membrane and the mass of fluid that must be displaced. By reducing the amount of optical fluid used, much faster tuning times could be achieved. As seen in Figure 19 , the lens membrane is not implanted, giving better optical transmission, and a larger tuning range.

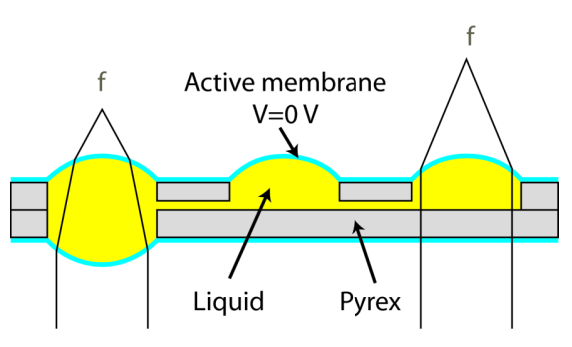

Figure 17: Schematic cross-section of the EAP tunable lens concept. Each tunable lens is coupled to an "active" device by an optical fluid. Devices thus operate in pairs.

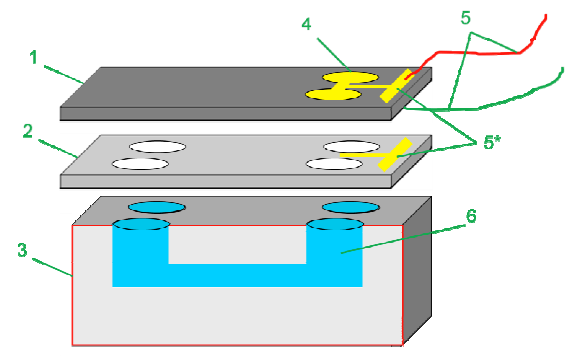

Figure 18: Simplified diagram of lens assembly, consisting of a bottom PDMS plate with fluidic channel (3), a Pyrex plate with hole (2) and the PDMS membrane (1) with implanted contacts.

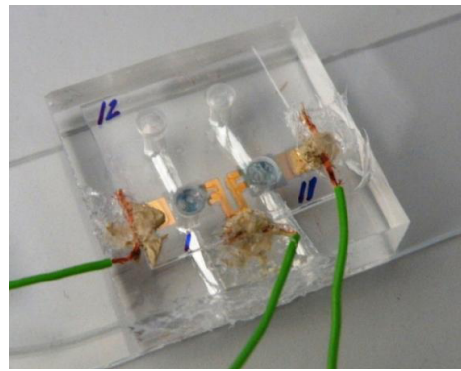

Figure 19: Photo of the tunable lens device shown in Figure 18. The two actuators are at the bottom (with electrical contacts), the two tunable lenses at the top. 


\section{2-axis steerable mirror}

As reported in the EAPAD 2011 proceedings ${ }^{[19]}$, our lab has recently made buckling mode actuators in which the circular top electrode is divided into three electrically isoalted parts, allowing more complex motion, as schematically illustrated in Figure 20. By mounting a mirror at the center of the membrane, 2-axis steering as well as up-down piston motion is possible.

One major challenge in miniaturizing such an actuator is avoiding electrical breakdown of the air in the $100 \mu \mathrm{m}$ gap between electrodes when they are biased to several $\mathrm{kV}$. It was therefore necessary to develop a multilayer approach, so that the region between the electrodes is filled with PDMS, which has a much higher dielectric breakdown strength than air. The multilayer approach also allows for larger force for a given voltage (in our case, twice the force since we have two layers). The membrane consists of two PDMS layers, each approximately $30 \mu \mathrm{m}$ thick, with three sets of ionimplanted electrodes, defined using laser-cut steel shadow masks.

The mirror (diced from a gold-coated silicon wafer) is mounted to the PDMS membrane using very small $(1 \mathrm{~mm}$ diameter) magnets. Another small magnet below serves to set the initial operating point. Two-axis tilting and up-down operation have been demonstrated for several membrane sizes ${ }^{[19]}$.

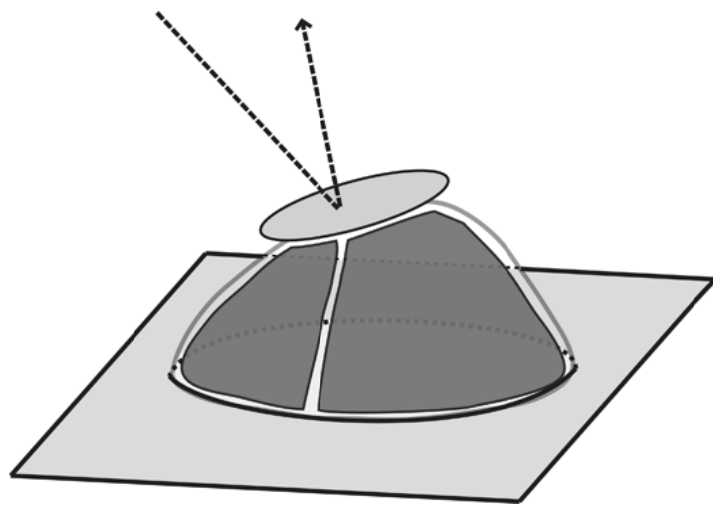

Figure 20: Schematic of a buckling membrane with the top electrode patterned into three individually controllable segments.

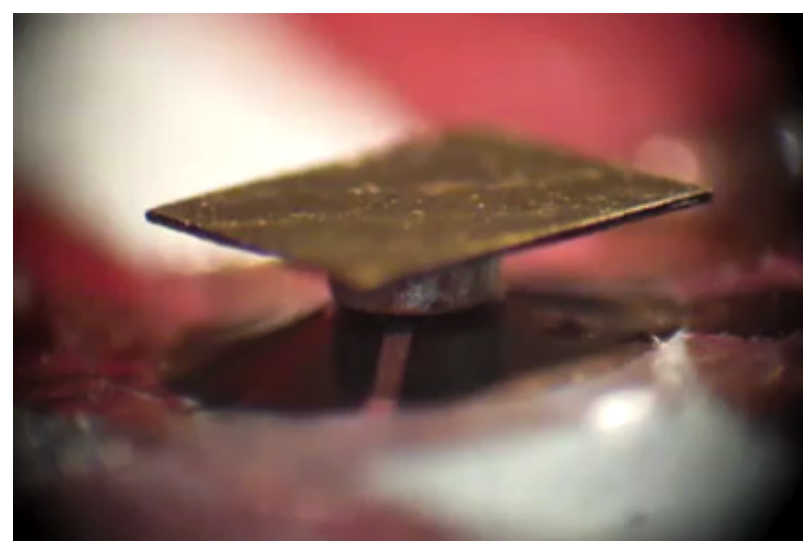

Figure 21: Photo of the multilayer steerable mirror device reported in [19]. Mirror dimensions are $3 \mathrm{~mm} \times 3 \mathrm{~mm}$. The magnet is visible as a post between the segmented ionimplanted PDMS membrane and the gold-coated mirror.

\subsection{Arrays of in-plane actuators}

The ion-implantation technique is not limited to making buckling-mode actuators. We describe in this section a device consisting of an array of 72 EAP devices, each $100 \mu \mathrm{m} \times 200 \mu \mathrm{m}$, to apply uniaxial strain to biological cells ${ }^{[17]}$.

Cell differentiation and gene expression are generally studied in vitro under static conditions (cells seeded onto rigid plastic substrates). Yet it is now recognized that cells modulate their behavior and developmental programs in response to mechanical stresses present in the organism. These include gravitational forces, tensile strains arising from bone elongation, compression forces within joints, or dynamic mechanical stimulation resulting from muscular activity. Notable examples of tissues whose development is regulated by mechanical input include skeletal, cardiac, and smooth muscles, bone, cartilage, tendons, the lungs and blood vessels. Investigation of these cell mechanotransduction mechanisms is currently performed with simple membrane cell-stretching equipment, relying on the distension of a flexible membrane. Uniaxial, biaxial, radial or circumferential strain can be applied, with strain of up to $20 \%$ at frequencies of order $1 \mathrm{~Hz}$, but the areas stretched are often in the range of centimeters, much too large to monitor singlecell responses; instead investigators often measure the averaged response of hundreds of thousands of cells that, by synchronizing their communal behavior, make it difficult to analyze the first stages of cell differentiation. 
Aschwanden et al.$^{[20]}$ developed at the ETHZ an EAP device to apply uniaxial strain to cells inside a cell culture liquid chamber, a compact system which was used by Thomasson et al. to study myoblasts ${ }^{[21]}$. The devices allow wide variation in strain and frequency while keeping the cells in a fixed focal plane during operation, but actuator size was of the $\mathrm{cm}$ scale.

The above was the motivation to make devices capable of applying mechanical strain to single cells. We therefore needed to make devices of the size of a cell (of order $100 \mu \mathrm{m}$ ), that can apply uniaxial strain of order $5 \%$ at speed of at least $1 \mathrm{~Hz}$, and that are bio-compatible.

The devices, shown schematically in Figure 22 and as realized in Figure 23, are fabricated by patterning compliant gold electrodes by ion-implantation on both sides of a $30 \mu \mathrm{m}$ thick PDMS film, which is bonded onto a Pyrex frame containing trenches. The top of the membrane is a continuous implanted electrode, while the bottom of the membrane has only narrow (100 $\mu \mathrm{m}$ wide) implanted electrodes. The active part of the device is where the implanted lines are suspended over a trench: at these intersections, the elastomer can be electrostatically compressed, leading to in-plane strain. The devices are electrically grouped to allow different strain levels or frequencies to be run simultaneously. The cells will be attached to each actuator following a surface treatment, and then cultured in an incubator.

Strain levels of $2 \%$ have been observed to date for voltages of $2 \mathrm{kV}$, and we are actively working to increase this to $10 \%$ by using other silicone materials and optimized electrodes. The cells are shielded from the high electric field in the actuated PDMS by using a grounded top electrode, and by grounding the conductive growth medium.

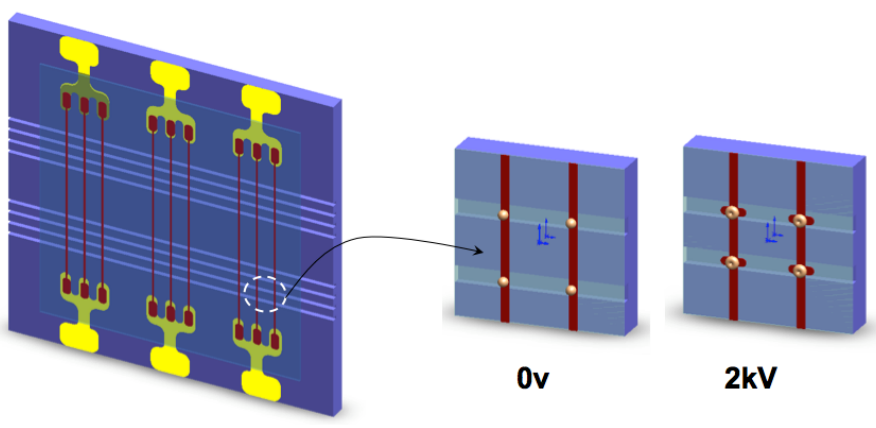

Figure 22: Simplified schematic of the cell-stretching chip. Red vertical lines are $100 \mu \mathrm{m}$ wide ion-implanted electrodes on the bottom side of a $30 \mu \mathrm{m}$ thick PDMS membrane. Horizontal lines are $100 \mu \mathrm{m}$ deep, $200 \mu \mathrm{m}$ wide trenches in the Pyrex chip. The membrane is bonded to the Pyrex, except over the trenches. There is a continuous (not visible here) implanted electrode on top of the membrane. The intersection of implanted lines and trenches form individual EAP actuators, 100x200 $\mu \mathrm{m}^{2}$.

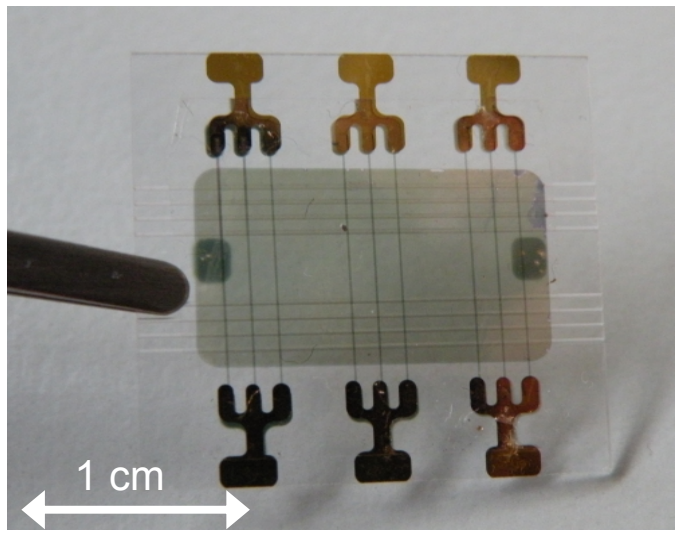

Figure 23: Chip containing an array of $72100 \times 200$ $\mu \mathrm{m}^{2}$ polymer actuators, each of which can apply uniaxial strain to a single muscle cell which will be attached to it.

\section{CONCLUSION}

Low-energy metal-ion implantation into PDMS can lead to highly compliant $20 \mathrm{~nm}$ thick electrodes, patternable on the $\mu \mathrm{m}$-scale, conducting at strains of over $150 \%$, that enable the microfabrication of arrays of miniaturized EAP actuators. The low stiffness and large maximum strain of the implanted electrodes allows for efficient operation of the chip-scale EAP devices. A variety of EAPs have been demonstrated using ion-implanted electrodes, including bi-directional buckling mode actuators, mm-scale tunable lens arrays, 2-axis beam steering mirrors, as well as arrays of 72 cell-size $\left(100 \times 200 \mu \mathrm{m}^{2}\right)$ actuators to apply mechanical strain to single cells. Current research includes complex arrays of micropumps and valves for lab-on-chip applications. 


\section{ACKNOWLEDGEMENTS}

This paper summarizes the research done on dielectric EAPs at the EPFL-LMTS between 2005 and 2010 by S. Akbari, P. Dubois, P. Moser, M. Niklaus, A. Punning, and S. Rosset. We thank Dr. Franco-Obregón for his collaboration on the cell stretchers. Financial support is gratefully acknowledged from: the Swiss National Science Foundation grants 200020-120164 and 200020-130453, from the Indo-Swiss Joint Research Programme (IJSRP) grant \#122948, and from the European Space Agency (ESA).

\section{REFERENCES}

[1] Ashley, S. “Artificial muscles,” Scientific American, vol. 289 (4), 52-59 (2003).

[2] Carpi, F., Rossi, D. D., Kornbluh, R., Pelrine, R., and Sommer-Larsen, P., Eds., [Dielectric Elastomers as Electromechanical Transducers], Elsevier, Amsterdam (2008).

[3] Brochu, P., Pei, Q., "Advances in Dielectric Elastomers for Actuators and Artificial Muscles", Macromol. Rapid Commun, 31, 10, (2010).

[4] Aschwanden, M., and Stemmer, A., "Low voltage, highly tunable diffraction grating based on dielectric elastomer actuators." Proc. SPIE 6524, 65241N-65241N-10 (2007)

[5] Pimpin, A., Suzuki, Y., and Kasagi, N., "Microelectrostrictive Actuator With Large Out-of-Plane Deformation for Flow-Control Application." Journal of Microelectromechanical Systems 16, no. 3, 753-764 (2007).

[6] Jordi, C., Michel, S., Dürager, C., Bormann, A., Gebhardt, C., Kovacs, G., "Large planar dielectric elastomer actuators for fish-like propulsion of an airship", Proc. SPIE 7642, 764223 (2010).

[7] Seker, E., Reed, M., Utz, M., and Begley, M.R., "Flexible and conductive bilayer membranes of nanoporous gold and silicone: Synthesis and characterization." Applied Physics Letters 92, no. 15, 154101 (2008).

[8] Urdaneta, M. G., Delille, R., and Smela, E., "Stretchable Electrodes with High Conductivity and PhotoPatternability." Advanced Materials 19, no. 18, 2629-2633 (2007).

[9] Rosset, S., Niklaus, M., Dubois, P., and Shea, H.R., "Mechanical Characterization of a Dielectric Elastomer Microactuator With Ion-Implanted Electrodes", Sensors and Actuators A, 144, pp.185-193, (2008).

[10] Dubois, P., Rosset, S., Koster, S., Stauffer, J., Mikhaïlov, S., Dadras, M., de Rooij, N., and Shea, H., "Microactuators Based On Ion-Implanted Dielectric Electroactive Polymer Membranes (EAP)", Sensors and Actuators A: Physical, Vol. 130-131, p. 147-154 (2006).

[11] Rosset, S., Niklaus, M., Dubois, P., and Shea, H.R., "Large Stroke Dielectric Elastomer Actuators with Ion Implanted Electrodes", Journal of Microelectromechanical Systems, 18, 1300 (2009).

[12] Dubois, P., Rosset, S., Niklaus, M., Dadras, M., Shea, H.R:, "Voltage Control of the Resonance Frequency of Dielectric Electroactive Polymer (DEAP) Membranes." Journal of Microelectromechanical Systems 17, no. 5 (2008).

[13] Rosset, S., Niklaus, M., Dubois, P., Shea, H.R., "Metal Ion Implantation for the Fabrication of Stretchable Electrodes on Elastomers", Advanced Functional Materials, Vol. 19 Issue 3, Pages 470 - 478, (2009).

[14] Niklaus, M., Rosset, S., Dubois, P., Dadras, M., and Shea, H., "Microstructure of $5 \mathrm{keV}$ Gold implanted Polydimethylsiloxane", Scripta Materialia 59 , pp. 893-896 (2008).

[15] Niklaus, M. and Shea, H., "Electrical conductivity and Young's modulus of flexible nanocomposites made by metal ion implantation of Polydimethylsiloxane: the relationship between nanostructure and macroscopic properties", Acta Materialia, vol. 59, pp .830-840, (2011).

[16]Choi, J.-W., Rosset, S., Niklaus, M., Adleman, J.R., Shea, H., Psaltis, D., "3-dimensional Electrode Patterning Within a Microfluidic Channel Using a Metal Ion Implantation”, Lab on Chip, vol. 10, p. 783-788, (2010).

[17] Akbari, S., Niklaus, M., Shea, H., "Arrays of EAP micro-actuators for single-cell stretching applications", Proc. SPIE - 7642, 76420H (2010).

[18] Niklaus, M., Rosset, S., and Shea, H., "Array of lenses with individually tunable focal-length based on transparent ion-implanted EAPs", Proc. of SPIE Vol. 7642, 76422K (2010).

[19] Punning, A., Akbari, S., Niklaus, M., Shea, H.R., "Mutlilayer Dielectric Elastomer Actuators with Ion Implanted Electrodes", Proc. SPIE Vol. 7976 (2011).

[20] Stemmer, A., Aschwanden, M., Franco-Obregón, Rey, A., Vonderheit, A., Enning, E., Sakai, M., "Bioreactor to apply mechanical forces as an anabolic stimulus ”, European Patent Number: EP 1990402 A1 (2008)

[21] http://www.lbb.ethz.ch/Publications/Posters/Cell_response_to_stretch_Thomasson.pdf 